\title{
Correspondence
}

\section{The BCL-2 database, Act 2: moving beyond dualism to diversity and pleiotropy}

\author{
A Aouacheria*,1 \\ Cell Death and Disease (2014) 5, e981; doi:10.1038/cddis.2013.511; published online 2 January 2014 \\ Subject Category: Cancer
}

Dear Editor,

BCL2 was discovered almost 30 years ago as part of a translocation $(\mathrm{t} 14 ; 18)$ in human B-cell lymphoma. ${ }^{1}$ Unique to all other oncogenes known to that date, the product of this gene turned out to act as a cytoprotective factor rather than as a cell growth promoter, ${ }^{2}$ providing a remarkable first in biology. The human BCL-2 protein was also the first identified component of the cellular machinery of apoptosis. Evolutionary conservation of this machinery became evident when it was realized that Caenorhabditis elegans was containing an antiapoptotic Bcl-2-like protein, CED-9. ${ }^{3}$ Soon thereafter, it also became clear that BCL-2 was belonging to a large group of proteins that can either repress (such as BCL-xL or MCL-1) or promote (e.g. BAX or BAK) apoptosis. The concept (first issued by Stanley Korsmeyer ${ }^{4}$ ) that the ratio between $B C L-2$ and $B A X$, and more generally between proapoptotic members and prosurvival members (the so-called 'rheostat' hypothesis), can determine cell survival or death after an apoptotic stimulus had a deep and sustained influence on the field. Most of the works performed on BCL-2 family proteins and most of the reviews written on the subject so far are rooted in this epistemological and ontological dualism, which has proven immensely useful in understanding the basic aspects of apoptosis regulation by $B C L-2$ family members. Yet, a number of studies have highlighted the need to transcend this simplistic dualism. These studies may be grouped into four main branches: (a) the demonstration that a distinct subclass of proapoptotic proteins sharing a single region of sequence similarity with BCL-2, termed the $\mathrm{BH} 3$ motif, act upstream of the various 'multi-BH' BCL-2 homologs; (b) evidence showing that at least certain BCL-2 proteins could be bi-functional, shifting from a cytoprotective to a cytotoxic activity and vice versa, depending on changes in the expression level, cell types, alternative splicing and post-translational modifications; (c) the findings that BCL-2 proteins, which act at the mitochondrial level to regulate apoptosis, could also exert functions at non-mitochondrial sites and, more importantly, could play physiological (non-apoptotic) roles in cells uncommitted to death, such as regulation of mitochondrial dynamics, metabolism and calcium homeostasis, or during development; ${ }^{5}$ (d) phylogenomics and bioinformatics studies documenting the important degree of divergence between BCL-2 homologous sequences, ${ }^{6}$ and the potential huge number of $\mathrm{BH}$-containing proteins (due to the poor definition of the $\mathrm{BH} 3$ signature). ${ }^{7}$ Collectively, these different points suggest that a major shift of paradigm is taking place in our understanding of BCL-2 proteins, moving from a notion of opposition (anti- versus proapoptotic members) to a notion of diversity (in sequence, structure, function, evolution) and pleiotropy (i.e., vital roles unrelated to cell death regulation). ${ }^{8}$

Taking this paradigm shift from a phylogenomics perspective, we have previously reported that duplications played a prominent role in the divergent evolution of BCL-2 homologous genes and contributed to the generation of substantial lineage-specific diversity in the BCL-2 complement of extant metazoans. ${ }^{6}$ Contrary to cellular BCL-2 homologous proteins, BH3-only proteins are evolutionarily unrelated to each other (i.e., they have not arisen from a common ancestor by duplication). Intriguingly, more than 30 cellular and viral proteins have recently been claimed to contain 'BH3-like' motifs, ${ }^{7}$ suggesting that the distribution of BH3-containing proteins might be broader than initially conceived. The large and heterogeneous mixture of proteins forming the contemporary BCL-2 group poses major challenges to researchers trying to investigate their structure-function relationships. As an attempt to help address this issue, we have built a novel version of the BCL-2 Database (http://bcl2db.ibcp.fr/). This novel version of BCL2DB represents an effort toward providing a more global picture of $\mathrm{BCL}-2$ proteins, with the objective of facilitating the reframe from dualism to multiplicity.

\section{Conflict of Interest}

The author declares no conflict of interest.

Acknowledgements. This work was supported by La Ligue Contre le Cancer (Comité de Saône-et-Loire) and Région Rhône-Alpes (CIBLE program). BCL2DB version 2 was developed by Valentine Rech de Laval, Professor Gilbert Deléage, Dr. Christophe Combet and the author on the PRABI platform funded by GIS IBISA-AO 2009. We wish to acknowledge and thank the past, present and

\footnotetext{
${ }^{1}$ Molecular Biology of the Cell Laboratory, Ecole Normale Supérieure de Lyon, LBMC UMR 5239 CNRS—UCBL-HCL-ENS Lyon, 46 Allée d'Italie, 69364 Lyon, France

${ }^{*}$ Corresponding author: A Aouacheria, Molecular Biology of the Cell Laboratory, Ecole Normale Supérieure de Lyon, LBMC UMR 5239 CNRS-UCBL—HCL-ENS Lyon, 46 Allée d'Italie, 69364 Lyon, France. Tel: + 334262359 43; Fax: + 334262359 01; E-mail: abdel.aouacheria @ens-lyon.fr
} 
future members of our Scientific Advisory Board for their thoughtful comments and suggestions.

1. Cleary ML, Smith SD, Sklar J. Cell 1986; 47: 19-28.

2. Vaux DL, Cory S, Adams JM. Nature 1988; 335: 440-442.

3. Hengartner MO, Horvitz HR. Cell 1994; 76: 665-676.

4. Korsmeyer SJ et al. Semin Cancer Biol 1993; 4: 327-332.

5. Hardwick JM, Soane L. Cold Spring Harb Perspect Biol 2013; 5: 2.

6. Aouacheria A, Brunet F, Gouy M. Mol Biol Evol 2005; 22: 2395-2416.
7. Aouacheria A et al. Trends Cell Biol 2013; 23: 103-111.

8. Ameisen JC. Cell Death Differ 2002; 9: 367-393.

(c) (i) (2)(2) Cell Death and Disease is an open-access journal published by Nature Publishing Group. This work is licensed under a Creative Commons Attribution-NonCommercialShareAlike 3.0 Unported License. To view a copy of this license, visit http://creativecommons.org/licenses/by-nc-sa/3.0/ 